\title{
Review
}

\section{Current Developments in LC-MS for Pharmaceutical Analysis}

\author{
Chang-Kee Lim* and Gwyn LoRD \\ MRC Bioanalytical Science Group, School of Biological and Chemical Sciences, Birkbeck, University of London; \\ Malet Street, London, WC1E 7HX, U.K. Received February 4, 2002
}

\begin{abstract}
The current developments in liquid chromatography-mass spectrometry (LC-MS) and its applications to the analysis of pharmaceuticals are reviewed. Various mass spectrometric techniques, including electrospray and nanospray ionization, atmospheric pressure chemical ionization and photoionization and their interface with liquid chromatographic techniques are described. These include high performance liquid chromatography, capillary electrophoresis and capillary electrochromatography and the advantages and disadvantages of each technique are discussed. The applications of LC-MS to the studies of in vitro and in vivo drug metabolism, identification and characterization of impurities in pharmaceuticals, analysis of chiral impurities in drug substances and high-throughput LC-MS-MS systems for applications in the "accelerated drug discovery" process are described.
\end{abstract}

Key words liquid chromatography-mass spectrometry; pharmaceutical analysis; drug metabolism study; drug discovery; impurities identification; high-throughput LC-MS-MS

Liquid chromatography-mass spectrometry (LC-MS) is an analytical technique that couples high resolution chromatographic separation with sensitive and specific mass spectrometric detection. This includes high performance liquid chromatography (HPLC)-MS, capillary electrophoresis (CE)-MS and more recently capillary electrochromatography (CEC)-MS. The technique is still fast developing, particularly in the mass spectrometry area, with vastly improved sensitivity and resolution. It is probably the most powerful technique currently available for pharmaceutical analysis.

This review is intended as a discussion on the current developments of LC-MS in pharmaceutical analysis rather than a review of literature alone. Various LC-MS techniques will be described briefly and the advantages and disadvantages of each will be discussed.

\section{DEVELOPMENTS IN LC-MS}

Brief History of LC-MS The last twenty years has seen a dramatic increase in the capabilities of MS. At the beginning of this period the invention of fast atom bombardment (FAB), by Barber et al. in 1981, ${ }^{1)}$ enabled easier analysis of involatile and thermally unstable molecules, especially those of biological interest. It may be argued that this technique acted as a catalyst for the development of other ionization techniques, such as matrix-assisted laser desorption/ionization (MALDI) and electrospray ionization (ESI), applicable to such molecules.

The combination of liquid chromatographic techniques with MS has been an important development. Early interfaces were concerned with coping with the potential of $11 / \mathrm{min}$ vapour in the MS ionisation source vacuum that would be generated by eluent from typical analytical columns flowing at $1 \mathrm{ml} / \mathrm{min}$. This was achieved by evaporating solvent on a heated moving belt, ${ }^{2,3)}$ or the use of very low flows, such as direct liquid introduction ${ }^{4,5)}$ and continuousflow FAB (CF-FAB). ${ }^{6}$ ) Thermospray $^{7)}$ and particle beam ${ }^{8)}$ interfaces were improved methods during the 1980s.

Atmospheric Pressure Ionization (ESI and APCI) Over the last decade, ESI and atmospheric pressure chemical ionization (APCI), have become the dominant techniques superseding thermospray etc. and are likely to remain so for the foreseeable future being inherently the most suitable for analytes in solution presented to the MS. ESI was originally proposed by Dole, ${ }^{9}$ who suggested using charged droplets as a source of ions for MS and Fenn ${ }^{10)}$ pioneered its development as an ionization source for MS, leading to the first commercially available instrument in 1989.

In essence, the electrospray process involves producing a fine spray of ionized droplets from the outlet of a capillary carrying a liquid stream, which may be eluent from HPLC, $\mathrm{CE}, \mathrm{CEC}$ or direct infusion of sample solution. This is achieved by applying a high voltage (typically $3-5 \mathrm{kV}$ ) to the outlet of the capillary at the spray tip, which creates a high electric potential and causes the production of a fine mist of droplets. This occurs at atmospheric pressure, hence the term atmospheric pressure ionization (API), is used. This process of nebulization may be aided by use of gas, usually nitrogen in pneumatically-assisted electrospray, called "ionspray" by the developers of this technique, ${ }^{11)}$ or by ultrasonic nebulization. ${ }^{12)}$ Nitrogen is also used as an aid to evaporate solvent from the droplets, which become smaller and hence the charge density increases until a point of instability is reached when the droplets break into smaller droplets. This process continues until de-solvated ions are produced, which pass into the high vacuum of the mass analyser through a small opening guided by electrical potential difference. A fuller explanation of the process is beyond the scope of this review, but a dedicated book $^{13)}$ and review ${ }^{14)}$ provide more information.

ESI is a "soft" ionization technique in that little fragmentation is produced, forming protonated or de-protonated ions respectively for positive and negative mode MS. Generally, negative mode is less sensitive, except where the nature of the analyte leads to the formation of stable anions, such as with carboxylic acids. An important feature of ESI is that multiply charged ions may be produced with large molecules, enabling their analysis in mass analysers with limited mass range. Mass analysers measure mass $(m)$ to charge $(z)$ ratio, $m / z$. In most ionization processes predominantly singly charged ions are produced where $z=1$ and $m / z=$ mass, but $z$ can be very large in ESI, especially for proteins. However, for molecules below about $1000 \mathrm{Da}$, then ESI produces predominantly singly charged ions usually. This would be the 
case for the vast majority of drug substances currently in use, but peptides and proteins are growing in importance in the pharmaceutical field.

The development of ESI led to a great increase in the use of LC-MS, it proving to be an efficient ionization technique, especially for polar compounds and very compatible with solvents used for reversed-phase HPLC. However, the early interfaces operated at flow rates in the low $\mu \mathrm{l} / \mathrm{min}$ range, which was too low for the typical $1 \mathrm{ml} / \mathrm{min}$ flow rates from analytical HPLC columns. Thus, it was necessary to split the flow or use capillary/microbore HPLC columns. The development of nebulization aids as mentioned above enabled higher flows to be accepted so that analytical columns could be connected directly with no splitting of eluent. In addition, aided nebulization produced better droplet formation with a wider range of solvents. Although eluent splitting is now strictly not necessary, it is often advantageous to split the flow from analytical columns or use capillary/microbore columns, keeping the ESI source cleaner and maintaining sensitivity. A further reason relates to the way ESI interfaces behave. Detectors in chromatography are of two main types, concentration-sensitive and mass-sensitive. ${ }^{15)} \mathrm{UV}$ detectors respond to changes in analyte concentration and are thus of the former type, while mass-sensitive detectors such as mass spectrometers respond to changes in the analyte mass flow. The response then is proportional to the absolute mass of analyte passing through the instrument, rather than concentration. There is an exception to this classification where mass spectrometers fitted with ESI sources behave essentially in a concentration-sensitive way. ${ }^{16)}$ Thus, smaller diameter HPLC columns show higher sensitivity when coupled with ESI-MS, since the analyte is more concentrated, eluting in a smaller volume of eluent. A further advantage of using smaller diameter columns and their low eluent flow rate, is that less sample, buffer and contaminants enters the mass spectrometer. Consequently, the ionization source remains cleaner for a longer period, maintaining sensitivity.

A development of ESI, APCI, produces ions by a corona discharge at atmospheric pressure. APCI is mainly applied to rather less polar compounds than ESI, up to about $1500 \mathrm{Da}$, generally producing singly charged ions and is able to accept flow rates of $1-2 \mathrm{ml} / \mathrm{min}$, utilizing a heated nebulizer to evaporate solvent. The technique is also less susceptible than ESI to ion suppression and has a wider dynamic concentration range, but is unsuitable for thermally unstable compounds. In addition, APCI can produce unexpected fragmentation, unlike ESI, which being a "soft" ionization method causes little fragmentation. "In-source" fragmentation can be induced by raising the sample cone voltage or MS/MS studies can be performed in a suitable instrument.

Microspray and Nanospray There have been further developments with ESI involving the use of lower liquid flows. Chowdhury and Chait in $1991^{17)}$ showed that a needle with a fine taper would allow aqueous solutions to be analyzed by ESI. The taper caused a higher electric potential to be produced at the capillary tip facilitating droplet formation and producing more efficient ionization and hence higher sensitivity. Several groups then pursued the development of low-flow interfaces, including Gale and Smith ${ }^{18)}$ and Emmett and Caprioli, ${ }^{19)}$ the latter using the term "micro-electrospray" to describe their sub $\mu \mathrm{l} / \mathrm{min}$ flow rate technique. Wilm and
Mann ${ }^{20,21)}$ used even lower flow rates of nanolitres per minute in their "nanospray" technique, employing capillaries with a tapered outlet of $1-2 \mu \mathrm{m}$ inner diameter (i.d.). This method was initially developed for the analysis of single protein samples deposited in the capillary, allowing electrospraying for about $20-30 \mathrm{~min}$ from $1 \mu \mathrm{l}$ of sample solution. This allowed ample time to carry out full scan and tandem MS/MS studies with very low sample consumption. This original description of nanospray was an off-line technique of course, but may equally be applied to on-line LC-MS.

The requirements for micro/nano-spray are a low i.d. capillary outlet, usually incorporating a tapered tip and electrical contact, which is usually via metal coating of the capillary tip, typically applied by gold deposition. Drawbacks with the technique include the need for a skilled operator and the metal tip coating is not robust, although it is possible to make electrical connection at a coupling prior to the tip.

Microfluidics On an even smaller scale, microfluidic chip devices were first reported for ESI-MS in $1997 .^{22-24)}$ These devices incorporated etched microchannels on glass chips from which solutions were electrosprayed from the microchannel exit. Separations such as CE and CEC can be performed on these chips within extended length channels, often with a serpentine configuration. Figure 1 shows a diagrammatic representation of a electrospray chip made by Lee et $a l^{25)}$ and incorporating needles at the channel exits for electrospray. The advantage of using such systems again relates to their possibilities in high throughput analysis and in automation. There are further developments in this area, including even the incorporation of a mass spectrometer on a chip. A recent short review on the topic provides further information. $^{26)}$

Matrix Effects Probably the widest application of LCESI-MS is in analysis of compounds such as pharmaceuticals in complex biological matrices. However, the presence of components in the matrix can suppress the analyte response, probably by competition for the ESI droplet surface and hence ionization. These effects can cause differences in response between sample in matrix and standards, leading to difficulties in quantitative analysis and compound identification. This emphasises the importance of the chromatographic step in the analysis, where good separation can reduce or eliminate these effects. It is interesting to note that it was thought that the invention of coupled MS/MS instruments

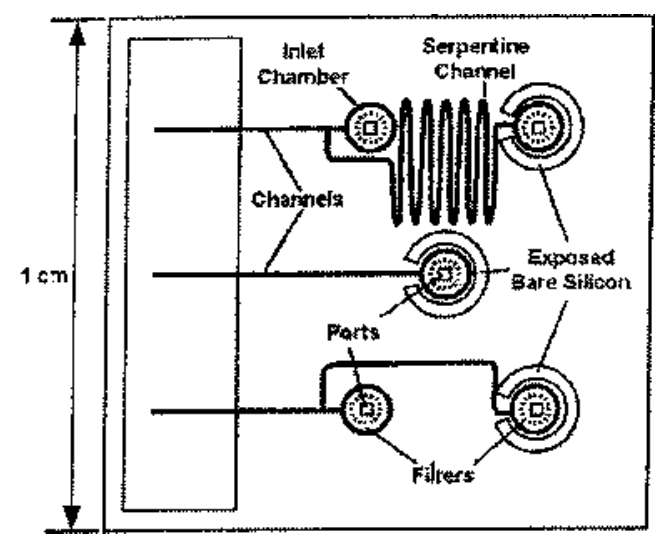

Fig. 1. Diagrammatic Representation of Electrospray Chip Adapted from ref. 25. 
would enable analysis of mixtures without separation, but suppression effects prevented this of course.

Generally, inorganic buffers should be avoided for ESI, since being involatile they accumulate in the ion source and preferentially ionize at the expense of the analyte. Ammonium acetate and formic acid are preferred to promote ionization and act as chromatographic buffers.

Trifluoroacetic acid (TFA) requires a particular mention. TFA and other volatile strong acids used as modifiers in HPLC are not usually compatible with ESI-MS, since they cause ion suppression. In positive ion mode, detection limits for basic compounds are reduced to about one-tenth compared to when acetic acid is used. ${ }^{27)}$ Signal suppression is caused by strong ion pairing between the TFA anion and the protonated analyte cation of basic molecules, effectively making them neutral. ${ }^{28)}$ In negative ion mode, complete suppression of analyte can occur by TFA competing for charge. ${ }^{29)}$ Aqueous solutions of TFA have high conductivity and surface tension, which causes spray instability in ESI and many methods have been reported to try to reduce these effects. For example, Kuhlmann et al. ${ }^{28)}$ used post column addition of propionic acid and isopropanol to improve positive mode ESI-MS. Isopropanol functioned to lower the surface tension, which showed only slight improvement in sensitivity, whilst propionic acid had a larger effect. The hypothesis is that propionic acid as a weaker acid competes with TFA for protons and because TFA is more volatile, it evaporates faster from the electrospray droplet. This results in the acid equilibrium being driven toward deprotonation of the weak acid as the droplet gets smaller and formation of a weak ion pair between analyte and the weak acid anion. This ion pair is sufficiently weak, compared to ion pairing with TFA, that the analyte ion may be ejected and hence sensitivity is improved. Other techniques for improvement of sensitivity include the recent report on the use of on-line membrane extraction of TFA for negative mode ESI-MS by New et al. ${ }^{29)}$ These techniques may find application where TFA is used in preference for substitution with a more MS compatible buffer such as ammonium acetate. TFA providing superior chromatographic resolution in many cases, especially for peptides and proteins.

Other Ionization Methods An ideal ionization source is one that ionizes all analytes with the same efficiency. However, suppression effects are a major obstacle in achieving this ideal situation.

A relatively new method of ionization for LC-MS is atmospheric pressure photoionization (APPI). ${ }^{30,31)}$ The mechanism for APPI is proton absorption and electron ejection from a molecule to form a charged molecular ion in the case of non-polar compounds. This process is independent of the presence of other molecules and does not suffer from ion suppression effects it is claimed. ${ }^{30)}$ In the presence of water or protic solvents, protonated molecule ions can be formed, as with ESI. A further development of the technique uses an ionizable dopant, whose ions react with analyte to improve analyte ionization and sensitivity. ${ }^{31)}$ In this case, similar behaviour to corona discharge APCI is shown, being suitable for compounds with high basicity or acidity in the gas phase. Thus, protonated or de-protonated ions are formed respectively. At present, the techniques have been applied to a limited range of test compounds and further demonstration of applicability to a range of real samples is needed to prove the worth of what seems to be a very interesting development.

Developments continue in coupling LC with other ionization techniques, for example, Cappiello et al. ${ }^{32)}$ describe two approaches for coupling electron ionization (EI), with capillary HPLC. The first method uses a micro-scale particle beam interface called "Cap-EI" and uses nitrogen to nebulize the liquid stream at $1-5 \mu \mathrm{l} / \mathrm{min}$. The second method involves interfacing into a modified EI source at lower flow rates of about $1 \mu \mathrm{l} / \mathrm{min}$. The advantage of using EI ionisation is the possibility of producing library searchable spectra for compound identification from suitable small/medium size molecules of low polarity. The technique is not as widely applicable as ESI, but could offer an additional useful method for analysis.

MALDI will not be discussed here, although being an important technique, it is not routinely connected to LC at present in a dynamic way, instead, fractions are collected for subsequent analysis. For example, HPLC peptide fractions collected on polymer membranes for later analysis by MALDI. ${ }^{33)}$ Gusev has recently reviewed interfacing MALDI with column and planar separations. ${ }^{34)}$

CE and CEC CE interfacing is now almost universally via ESI and nanospray is ideal, since it operates at flow rates typical of those produced in CE. Conventional ESI operates at higher flow rates, necessitating a "make-up" flow to about $5 \mu \mathrm{l} / \mathrm{min}$ and this is best provided via a co-axial system, as originally described by Smith's group. ${ }^{35)}$ The make-up liquid is typically an organic solvent such as methanol, facilitating more efficient spray production when mixed at the capillary tip with the usually aqueous CE eluent. Developments in CE/MS have been reviewed. ${ }^{36}$

CEC utilizes the same interfaces as CE, as may be expected, being very similar techniques. CEC uses capillaries, typically $50-100 \mu \mathrm{m}$ i.d., packed with HPLC stationary phases. Flow of eluent is achieved by electroosmotic flow, rather than hydrodynamic pumping as in HPLC and separation may be considered a mixture of CE and HPLC partition mechanisms. The first coupling of CEC with MS was by Verheij et al. ${ }^{37)}$ who used pressure assistance for eluent flow in the technique known as "pseudoelectrochromatography" and CF-FAB-MS. True CEC with pure electroosmotic flow was also first coupled to CF-FAB-MS, ${ }^{38)}$ but virtually all subsequent reports have used ESI. A review ${ }^{39)}$ and a dedicated book $^{40)}$ provide further information.

As noted in the section on matrix effects, the use of inorganic buffers that are common in CE/CEC are not desirable and suitable alternatives should be used if possible. However, because the flow rates are so low in these techniques, then a limited amount of inorganic buffer can be tolerated, especially when diluted by a make-up liquid.

Mass Analyzers Developments in time-of-flight (TOF) analysers have been rapid over the last few years, particularly because of developments in digital electronics. The resurgence of interest in TOF analysers probably arose from their use in MALDI. Initially, TOF was a low resolution technique, but is now capable of 20000 plus, resolution, largely because of the use of a reflectron. This resolution allows "exact mass" measurements to be made on small/medium size molecules, from which empirical formula can be determined. Other advantages of TOF analysers include fast spec- 


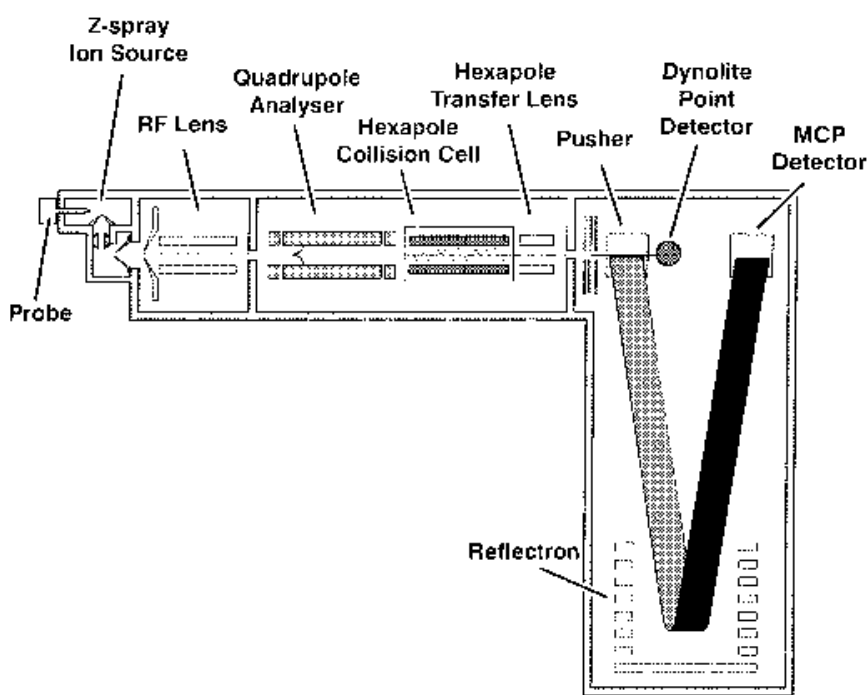

Fig. 2. Diagrammatic Representation of Q-TOF Mass Spectrometer with "Z-Spray" (Micromass)

tral acquisition, accuracy in mass measurement arising from stability over the $\mathrm{m} / \mathrm{z}$ range and no theoretical limit to $\mathrm{m} / \mathrm{z}^{41}$ ) The use of TOF instruments is likely to continue to increase for the foreseeable future, superseding the use of magnetic sector instruments in many areas.

Pharmaceutical compound analysis in the recent past has been dominated by the use of quadrupole and to a much lesser extent, ion-trap instruments, coupled with API sources. This was due to their relatively low cost, small size, mass range and reasonable scanning speed. Coupled tandem MS/MS quadrupole systems in particular have provided structural information and highly selective detection and quantification of specific metabolites by improvements in signal to noise. However, ESI-TOF instruments are now becoming more prominent in drug analysis, especially when coupled with a quadrupole analyzer as a Q-TOF tandem MS/MS instrument. In addition to the advantages of TOF analyzers noted before, the resolution of a Q-TOF instrument is improved by ion focussing in the quadrupole, although mass range is reduced, but duty cycle is also reduced. Figure 2 shows a diagrammatic representation of a Q-TOF instrument fitted with an orthogonal ESI source.

A Q-TOF instrument allows exact mass information to be obtained on both product and precursor ions in a tandem MS/MS experiment, improving analytical capability. Comparison of the performance of a Q-TOF with a Quadrupole MS/MS instrument for analysis of a drug in plasma, showed the former better in overcoming interference from matrix by more accurate mass measurements. ${ }^{42}$

TOF analyzers are able to acquire spectra at a very high rate, in the microsecond range, resulting in high sensitivity and the ability to acquire sufficient data from very narrow chromatographic peaks. Quadrupole only analyzers show much lower sensitivity because they scan (as do magnetic sector analyzers) and spend very little time at any particular $\mathrm{m} / \mathrm{z}$ value. For this reason they show highest sensitivity, similar to a TOF instrument, in selected ion monitoring (SIM) or selected reaction monitoring (SRM) modes, particularly relevant for quantitative work. In acquiring product ion spectra, the Q-TOF instrument is $10-100$ times more sensitive than a quadrupole MS/MS instrument, measuring samples in the femtomole range. ${ }^{43}$

High throughput analysis with fast chromatography produces very narrow chromatographic peak widths of only a few seconds, as does CE, but a TOF instrument can acquire spectra fast enough to determine them at high accuracy. This obviates, to an extent, the inherent problem of poor chromatographic efficiency.

Fourier transform-ion cyclotron resonance (FT-ICR), a technique of very high mass resolution will not be discussed in any depth, at present, instrumentation is large, complex and expensive. However, with advances in technology it will become much more prominent in the future, especially in the field of proteomics, for example, capillary HPLC has been coupled to FT-ICR via ESI for proteomics. ${ }^{44,45)}$ The technique has a useful mass range of about 10000 with capability of 1 million resolution.

LC-MS Instrumentation Many instrument manufacturers now offer combined, integrated HPLC/MS systems, especially resulting from recent HPLC and MS instrument company mergers. However, it is often desirable for the user to purchase separate instruments, to achieve the best performance. The only drawback with this is that if instruments are purchased from different manufacturers, then software may not be compatible. However, this is becoming less of a problem, as software is available from some manufacturers that will operate instruments from others, especially MS operating systems that can also control several different types of HPLC system.

ESI sources are now usually orthogonal to the analyzer, rather than in-line, resulting in the maintenance of high performance by reduction of source contamination, for example, the "Z-Spray" ESI source by "Micromass" (Micromass Ltd., Manchester, U.K.).

The pharmaceutical industry over the last ten years has made increasing demands for faster analytical methods, particularly because of the high sample throughput generated by combinatorial chemistry. LC/MS has been the most prominent technique, with its high sensitivity and specificity. Very fast LC gradients of only a few minutes are now used on short columns (typically $3-5 \mathrm{~cm}$ long), compared to separations previously on $25 \mathrm{~cm}$ long columns with $30-60 \mathrm{~min}$ gradients. However, there are limitations of using short columns with fast gradients where chromatographic separation is likely to be incomplete. Suppression effects mentioned above can be a significant problem.

Samples are also analyzed without any separation by flow injection analysis (FIA), directly into the MS, but again there are inherent problems of ionization suppression.

Fraction collection coupled with LC/MS has also recently been introduced by several manufacturers. These are similar to the previously available systems where selected fractions are collected by their response on a UV detector. For MS, the expected mass triggers collection of a fraction from the eluent at a split, prior to the MS. UV detection in parallel with MS also provides further structural information and indication of purity and may display components not observed on the MS (because of inability to ionize or ion suppression).

With the advent of fast acquisition TOF MS, it has become possible to maximize sample throughput with multiple inlet systems into one MS to make most economic use of a high 
cost instrument. De Biasi et al. $^{46)}$ have described the use of four HPLC columns in parallel coupled to one ESI-TOF instrument. The MS was fitted with a four-channel multiplex ESI source, in which each of the four liquid streams is sampled for 0.1 seconds, resulting in the production of individual mass spectra synchronized to each HPLC column. This system has been developed commercially by "Micromass" (Micromass Ltd., Manchester, U.K.) and coupled to their MS instruments. Further developments include use of an eightchannel multiplex ESI source and of particular note is the availability of a dual ESI inlet system. ${ }^{47)}$ This has been developed for accurate mass work where the sample and calibrant may be analyzed in rapid succession and improve accuracy of mass measurements. Another approach ${ }^{48}$ uses three switching valves and four HPLC columns for staggering injections onto the columns and allowing the mass spectrometer to continuously analyze the chromatographic window of interest. Using this approach, the optimized run time is slightly greater than the sum of the widths of the desired peaks.

Combinatorial chemistry has also led to the use of 96-well microtitre plates and their coupling with analytical systems. For example, Liu et al. ${ }^{49)}$ used a multichannel device with an array of 96 electrospray tips for MS analysis, with potential to analyze 720 samples per hour.

The result of this improved ability to analyze many more samples in a given time and faster acquisition MS instruments is the production of large amounts of data. This is becoming a major issue and improvements in data management are needed utilizing automated processing and interpretation.

\section{APPLICATIONS OF LC-MS}

The major applications of LC-MS in pharmaceutical analysis have been in drug metabolism studies, the analysis and identification of impurities and degradation products in pharmaceuticals and the isolation and characterization of potential drug substances from natural or synthetic sources.

LC-MS in Drug Metabolism Studies The metabolism of a potential new pharmaceutical must be studied before it can be considered for further development into a therapeutic agent. A good drug should ideally be metabolically stable and show a good pharmacokinetic profile with high bioavailability and long half-life. Some metabolites may also be more pharmacologically active or more toxic than the parent drug. Characterization of the major and active metabolites helps in the discovery and design of new drug candidates with improved pharmacological activity, metabolic stability and toxicology profile.

LC-MS is the method of choice for the study of drug metabolism because of its sensitivity and specificity. It also provides molecular weight information and fragmentation patterns for structure elucidation. Which ionization technique is used will depend on the structure and the acidity or basicity of the drug studied. In general, positive or negative ion ESI is preferred for strongly basic or acidic drugs respectively, while APCI is more suited for the less basic/acidic or neutral molecules.

In Vitro Drug Metabolism The liver is the primary organ that metabolizes drugs. Preliminary studies of drug metabolism have therefore commonly been carried out in

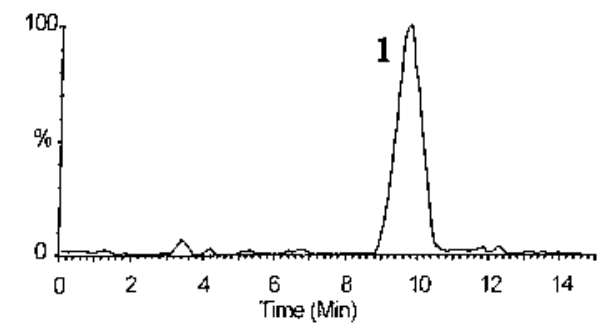

mis 374

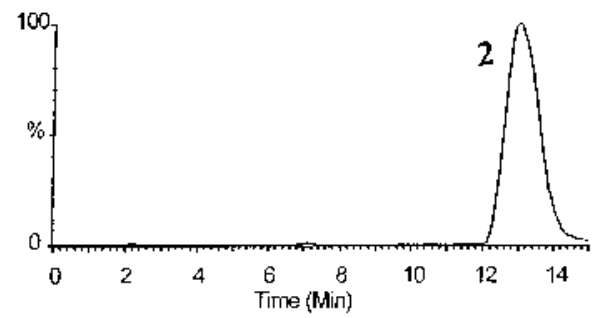

$m / 238$

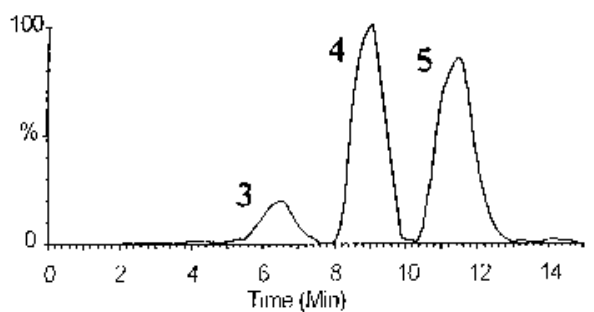

Fig. 3. HPLC-ESI-MS Separation of Droloxifene and Its Metabolites

Droloxifene was incubated with human liver microsomes for $30 \mathrm{~min}$ and the resulting metabolites separated by isocratic HPLC on a Hypersil-ODS column ( $5 \mu \mathrm{m}$ particle size, $250 \times 4.6 \mathrm{~mm}$ i.d.) with $70 \%$ methanol in $0.5 \mathrm{~m}$ ammonium acetate as mobile phase at a flow rate of $1 \mathrm{ml} / \mathrm{min}$. Detection was on-line (1:9 split), using a Micromass Quattro II ESI-MS in positive ion mode. Peaks: $1, N$-desmethyldroloxifene $(m / z 374) ; 2$, droloxifene $(\mathrm{m} / \mathrm{z} 388) ; 3, \alpha$-hydroxydroloxifene $(\mathrm{m} / \mathrm{z} 404) ; 4$, 4-hydroxydroloxifene $(\mathrm{m} / \mathrm{z} 404)$; 5 , droloxifene $N$-oxide $(\mathrm{m} / \mathrm{z} 404)$.

vitro with liver microsomal preparations or hepatocytes. ${ }^{50-52)}$ These provide good initial indication of the metabolic fate of a drug.

The metabolism of droloxifene, ${ }^{53)}$ an analogue of the antibreast cancer drug tamoxifen, by human liver microsomes is an example of the application of LC-MS in an in vitro metabolism study. The HPLC-ESI-MS mass chromatogram is shown in Fig. 3. The metabolites identified (Fig. 4) are $N$ desmethyldroloxifene $\left[(\mathrm{M}+\mathrm{H})^{+}, m / z\right.$ 374], 4-hydroxydroloxifene $\left[(\mathrm{M}+\mathrm{H})^{+}, m / z 404\right]$, droloxifene $N$-oxide $\left[(\mathrm{M}+\mathrm{H})^{+}\right.$, $m / z 404]$ and $\alpha$-hydroxydroloxifene $\left[(\mathrm{M}+\mathrm{H})^{+}, m / z\right.$ 404]. The example clearly demonstrates the importance of chromatographic resolution in LC-MS analysis. There are three metabolites with the $(\mathrm{M}+\mathrm{H})^{+}$ion at $m / z 404$ and the unequivocal identification of these metabolites is difficult without complete separation.

In Vivo Drug Metabolism In vivo metabolism studies involve analysis of drugs and metabolites in blood, urine and faeces. These samples contain a larger amount of endogenous compounds that could co-elute and interfere with the LC-MS analysis. A good sample preparation technique coupled with efficient chromatographic separation is therefore essential for the successful application of LC-MS to in vivo metabolism studies.

Figure 5 shows the HPLC-ESI-MS mass chromatogram of tamoxifen and its metabolites from the plasma of a breast cancer patient treated with tamoxifen. There are three peaks with the $(\mathrm{M}+\mathrm{H})^{+}$ion at $\mathrm{m} / \mathrm{z} 388$. These are 4-hydroxytamox- 


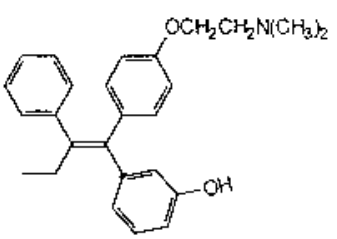

I

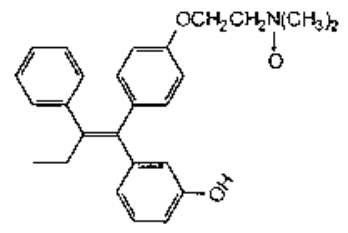

[I]
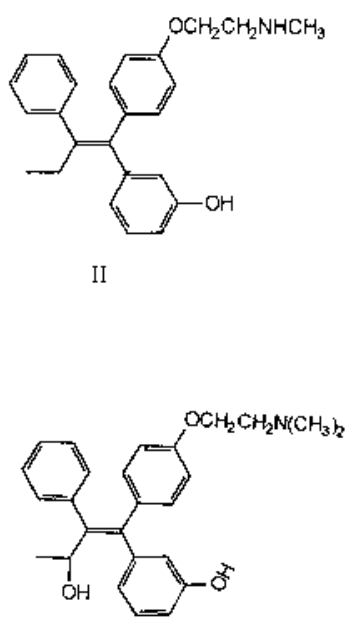

$\left[Y^{\prime}\right.$

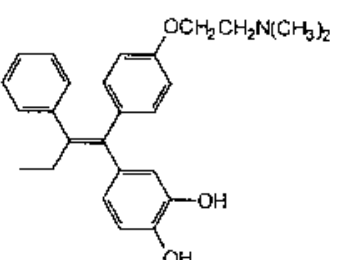

$\mathrm{V}$

Fig. 4. Structures of Droloxifene and Metabolites Detected in Human Microsomal Incubation

I, droloxifene; II, $N$-desmethyldroloxifene; III, droloxifene $N$-oxide; IV, $\alpha$-hydroxydroloxifene; V, 4-hydroxydroloxifene.

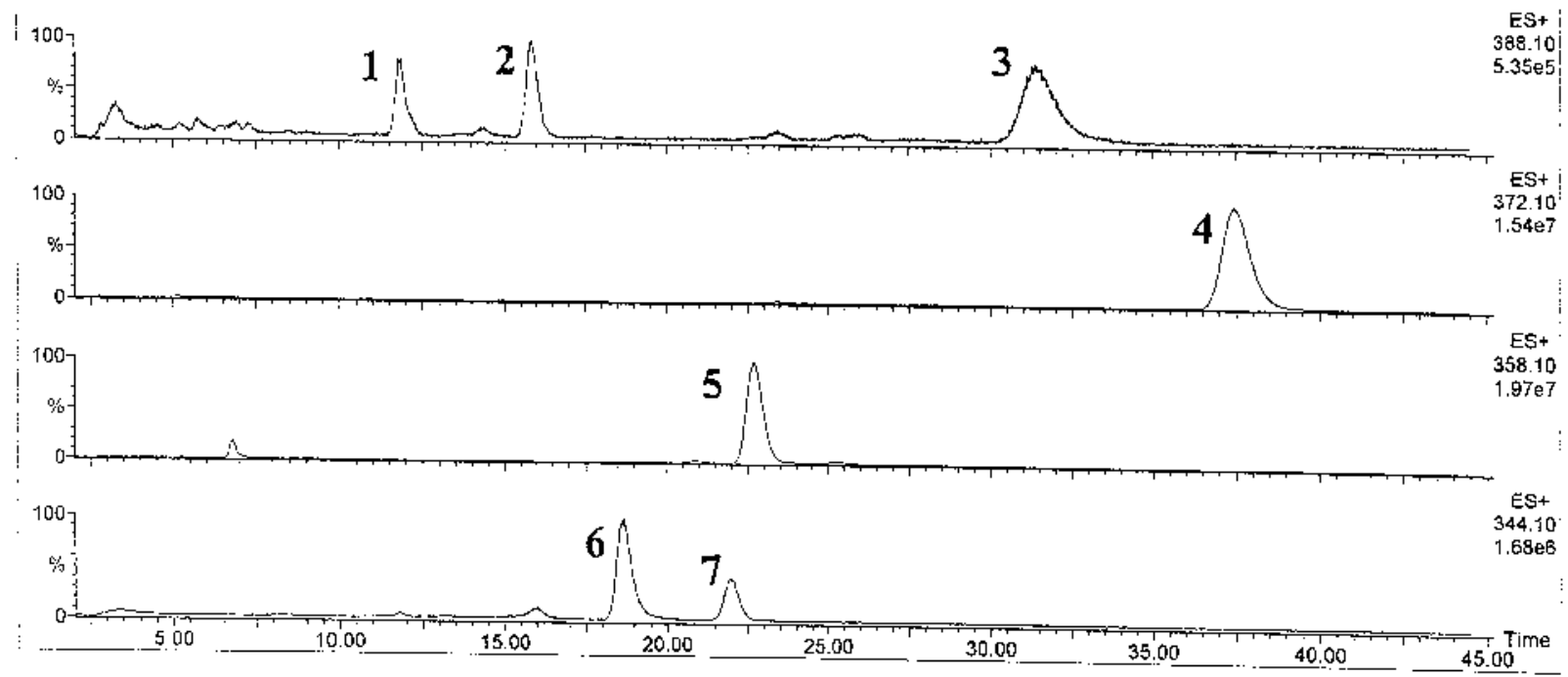

Fig. 5. HPLC-ESI-MS Separation of Tamoxifen and Metabolites in the Plasma of a Patient on Tamoxifen Therapy

The separation was carried out on a Hypersil-ODS column ( $5 \mu \mathrm{m}$ particle size, $250 \times 4.6 \mathrm{~mm}$ i.d.) with $70 \%$ methanol in $0.5 \mathrm{M}$ ammonium acetate as mobile phase at a flow rate of $1 \mathrm{ml} / \mathrm{min}$. Detection was on-line (1:9 split), using a Micromass Quattro II ESI-MS in positive ion mode. Peaks: 1 , 4-hydroxytamoxifen; 2, 4'-hydroxytamoxifen; 3, tamoxifen $N$ oxide; 4, tamoxifen; 5, $N$-desmethyltamoxifen; 6 , endogenous plasma component; 7, $N$-desdimethyltamoxifen.

ifen (peak 1), 4'-hydroxytamoxifen (peak 2) and tamoxifen $\mathrm{N}$-oxide (peak 3$)$. Peaks $4(\mathrm{~m} / \mathrm{z} 372)$ and $5(\mathrm{~m} / \mathrm{z} 358)$ are tamoxifen and $N$-desmethyltamoxifen, respectively (Fig. 6). There are also two peaks with the $(\mathrm{M}+\mathrm{H})^{+}$ion at $\mathrm{m} / z 344$. Tandem MS/MS analysis showed that peak 6 is an endogenous plasma component not related to tamoxifen and peak 7 is $\mathrm{N}$-desdimethyltamoxifen with a characteristic $\mathrm{CH}_{2} \mathrm{CH}_{2} \mathrm{NH}_{2}$ $(m / z 44)$ fragment. Tamoxifen and its derivatives all share a common characteristic fragmentation pathway ${ }^{54,55)}$ of sidechain cleavage to give ions at $\mathrm{m} / z 72,58$ or 44 corresponding to $\mathrm{CH}_{2} \mathrm{CH}_{2} \mathrm{~N}\left(\mathrm{CH}_{3}\right)_{2}, \mathrm{CH}_{2} \mathrm{CH}_{2} \mathrm{NHCH}_{3}$ or $\mathrm{CH}_{2} \mathrm{CH}_{2} \mathrm{NH}_{2}$, respectively. Tandem MS/MS thus allows the positive identification of a tamoxifen metabolite.

Figure 7 shows an example of the application of negative ion ESI-MS in in vivo metabolism studies; the identification of curcumin metabolites in rat plasma after administration of curcumin via the i.v. route. ${ }^{56}$ Curcumin and its derivatives are phenolic compounds and difficult to detect by positive ion ESI-MS. Three metabolites have been positively identified. These are hexahydrocurcumin $\left(\mathrm{M}-\mathrm{H}^{-}, \mathrm{m} / \mathrm{z} 373\right)$, hexahydrocurcuminol $\left[(\mathrm{M}-\mathrm{H})^{-}, m / z\right.$ 375] and hexahydrocurcumin glucuronide $\left[(\mathrm{M}-\mathrm{H})^{-}, m / z\right.$ 549]. The $(\mathrm{M}-\mathrm{H})^{-}$ion for curcumin is at $m / z 367$. Tandem MS/MS was used for the characterization of the metabolites. Hexahydrocurcumin glucuronide, for example, gave a characteristic $(\mathrm{M}-\mathrm{H})^{-}$ion at $\mathrm{m} / \mathrm{z} 177$ corresponding to a fragment from the conjugated glucuronic acid. ${ }^{56)}$

High-Throughput LC-MS Analysis of Drugs and Metabolites There has been significant interest in "accelerated drug discovery" brought about by the development of combinatorial chemistry where hundreds of thousands of compounds can be generated within a short time. This led to the need for high-throughput methods of analysis not only during the reaction development, optimization and produc- 


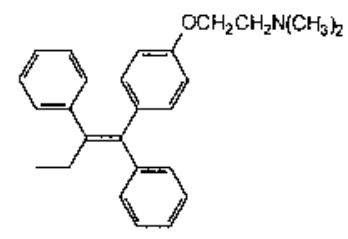

I

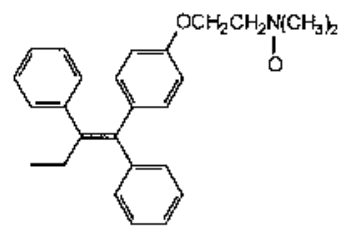

IV

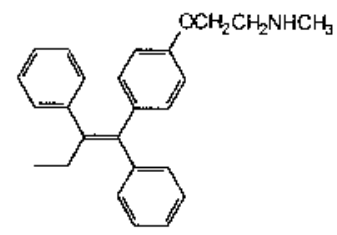

II

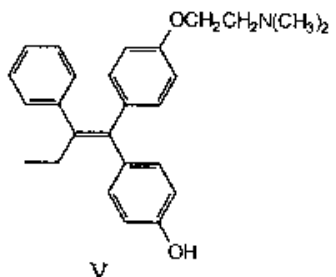

$\mathrm{V}$

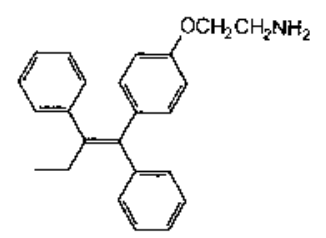

III

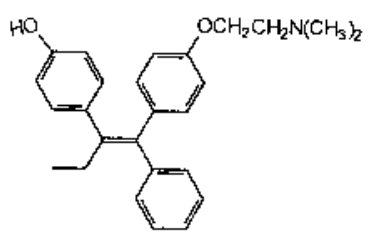

VI

Fig. 6. Structures of Tamoxifen and Metabolites Detected in the Plasma of a Patient on Tamoxifen Therapy

I, tamoxifen; II, $N$-desmethyltamoxifen; III, $N$-desdimethyltamoxifen; IV, tamoxifen $N$-oxide; V, 4-hydroxytamoxifen; VI, 4'-hydroxytamoxifen.
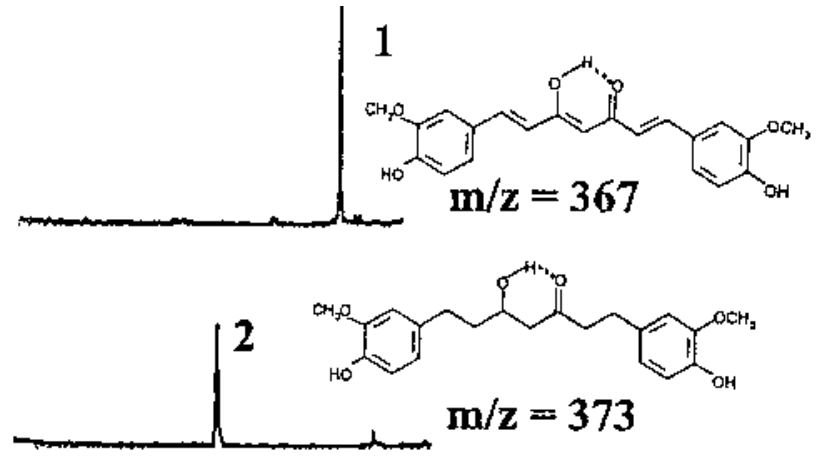

3

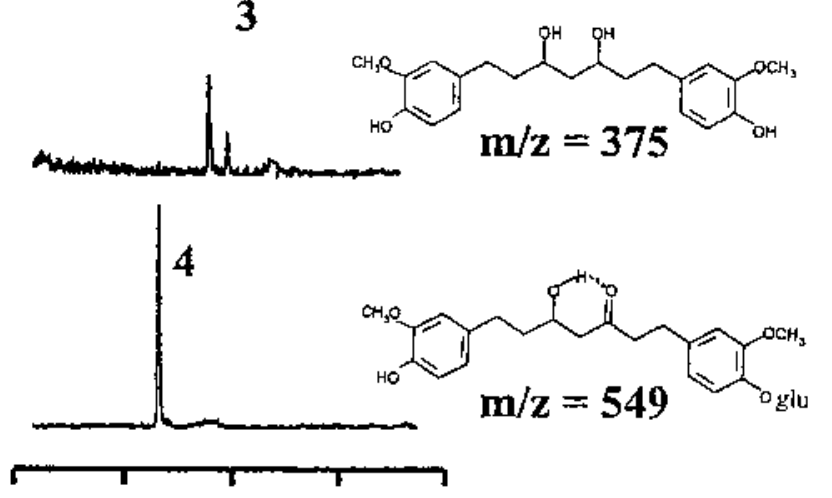

$\begin{array}{lllll}0 & 20 & 40 & 60 & 80\end{array}$

Retention time (min)

Fig. 7. HPLC-ESI-MS Separation of Curcumin and Metabolites in Rat Plasma $30 \mathrm{~min}$ after Administration of Curcumin $(40 \mathrm{mg} / \mathrm{kg}$, i.v.)

The separation was carried out on a Symmetry Shield RP18 column $(3 \mu \mathrm{m}$ particle size, $150 \times 3.9 \mathrm{~mm}$ i.d.) by gradient elution from 5 to $45 \%$ acetonitrile in $0.001 \mathrm{M}$ ammonium acetate $(\mathrm{pH} 4.5)$ in $60 \mathrm{~min}$, followed by an increase to $95 \%$ acetonitrile for a further $20 \mathrm{~min}$ at a flow rate of $1 \mathrm{ml} / \mathrm{min}$. Detection was on-line (1:9 split), using a Micromass Quattro II ESI-MS in negative ion mode.

tion stages, but also in the biological screening stage where drugs and metabolites are analysed in in vitro and in vivo biological samples. ${ }^{57)}$ High-throughput approaches to sample preparation coupled with LC-MS greatly improved the speed and sensitivity of analysis necessary for the accelerated drug discovery process.

The two main methods of high-throughput sample preparation are based on the use of automated 96-well extraction procedures. This can be in the solid phase extraction $(\mathrm{SPE})^{58-62)}$ or liquid-liquid extraction ${ }^{63-68)}$ format. The principles, operation procedures and applications of these methods have been described. ${ }^{58-68)}$ In these methods, a robotic liquid handling system is used to handle and process samples automatically, thus eliminating the time consuming manual steps in sample preparation.

High-throughput sample preparation is sometimes coupled with fast LC separation on short columns with on-line tandem MS/MS for the resolution of co-eluting peaks to further shorten the analysis time. While this technique may work for relatively clean extracts with few components, it is always advisable to achieve complete separation of analytes, as demonstrated in the analysis of droloxifene and tamoxifen metabolites above. Tandem MS/MS may assist with the identification of overlapping peaks of different structures, but not necessarily isomers. Matrix effects such as the suppression and modification of ionization by co-eluting endogenous components $^{69-71)}$ must also be considered.

Fast-flow on-line extraction methods based on the column switching technique have been described. ${ }^{72-76)}$ Biological samples, e.g. plasma, are injected directly into the LC-MS system without prior sample preparation. A narrow-bore HPLC column packed with large particle size material is used to extract small molecule analytes but allows unretained large molecules such as plasma proteins to flow to waste at high mobile phase flow-rate. The adsorbed analytes are then eluted through a column-switching valve into an analytical column for LC-MS/MS analysis. Very fast analysis can be achieved with this method. Again, care should be taken to ensure that there is no matrix effect in this high-throughput LC-MS/MS system. An LC-MS system that does not compromise on chromatographic separation but is capable of analyzing several samples in parallel, as in multiplexed LCMS-MS systems, would increase the efficiency of the use of 
the mass spectrometer and decrease the possibility of introducing matrix effects.

Analysis and Identification of Impurities and Degradation Products in Pharmaceuticals Drug Regulation Authorities require the purity of a pharmaceutical to be fully defined and the presence of impurities be fully tested and evaluated. This is important to ensure that the observed pharmacological and toxicological effects are truly those of the pharmaceutical and not due to the impurities. Analysis and monitoring of impurities and degradation products in formulated pharmaceuticals are essential for ensuring that no compounds with deleterious effects are generated during their shelf life. The identification of degradation products will aid in the understanding of potential side effects associated with degradation and in the design of a more favourable formulation and synthesis of new drugs with greater stability.

Impurities present in pharmaceuticals are mainly formed during the synthetic process from raw chemicals, intermediates, solvents and by-products. Raw chemicals and intermediates for drug manufacturing do not have the same purity requirements as the final pharmaceuticals. They are likely to contain components that affect the purity of the final manufactured pharmaceutical. Solvents used in the synthesis may also contain impurities that could react to generate pharmaceutical impurities. By-products are often generated during synthesis and are another source of pharmaceutical impurities.

Monitoring and controlling the penultimate step of pharmaceutical synthesis is crucial for the production of a relatively pure drug and the identification of any potential impurities present.

Impurities in pharmaceuticals may also arise from the ingredients used in dosage formulation and/or in the process of formulation where temperature, humidity and light may all play a part.

Drug degradation is often complex and unpredictable. The most common degradation processes are oxidation, hydrolysis and dehydration; Other processes include adduct formation with excipients, dimerization and rearrangement.

The International Conference on Harmonization (ICH) of Technical Requirements for Registration of Pharmaceuticals for Human Use has published guidelines for the reporting, identification and qualification of impurities in pharmaceuticals (ICH Q3A Impurities in new drug substances and $\mathrm{ICH}$ Q3B Impurities in new drug products, October 1999). The thresholds for reporting of unknown impurities and identification and qualification of impurities and degradants in new drugs are shown in Table 1.

Since impurities and degradants are usually present in relatively small quantities compared to the drug, an analytical technique capable of separating a mixture containing highly varied concentrations of analytes with sensitive and specific detection is required. LC-MS is therefore widely used for this purpose. ${ }^{77-81)}$

However, it is useful to use an UV-Visible detector in conjunction with mass spectrometry for the estimation and identification of impurities, especially for the unknowns. The UVvisible absorption and response factor of related compounds tend to be similar, while their MS ionization efficiencies can be significantly different.

Figure 8 shows the HPLC separation of impurities and
Table 1. Thresholds for Reporting, Identification and Qualification of Impurities and Degradants in Pharmaceuticals

\begin{tabular}{|c|c|c|c|}
\hline $\begin{array}{l}\text { Maximum } \\
\text { daily dose }\end{array}$ & Reporting & Identification & Qualification \\
\hline \multicolumn{4}{|l|}{ Drug substance } \\
\hline$\leq 2 \mathrm{~g} / \mathrm{d}$ & $0.05 \%$ & $0.1 \%$ or $1 \mathrm{mg} / \mathrm{d}$ & $\begin{array}{l}0.1 \% \text { or } 1 \mathrm{mg} / \mathrm{d} \\
\text { Intake (whichever } \\
\text { is lower) }\end{array}$ \\
\hline$>2 \mathrm{~g} / \mathrm{d}$ & $0.03 \%$ & $0.05 \%$ & $0.05 \%$ \\
\hline \multicolumn{4}{|l|}{ Drug product } \\
\hline$<1 \mathrm{mg}$ & $1 \%$ or $5 \mu \mathrm{g} / \mathrm{d}$ & $1 \%$ or $5 \mu \mathrm{g} / \mathrm{d}$ & \\
\hline $1-10 \mathrm{mg}$ & $0.5 \%$ or $20 \mu \mathrm{g} / \mathrm{d}$ & $0.5 \%$ or $20 \mu \mathrm{g} / \mathrm{d}$ & \\
\hline$<10 \mathrm{mg}$ & & & $1 \%$ or $50 \mu \mathrm{g} / \mathrm{d}$ \\
\hline $10-100 \mathrm{mg}$ & & & $0.5 \%$ or $200 \mu \mathrm{g} / \mathrm{d}$ \\
\hline$>10 \mathrm{mg}-2 \mathrm{~g}$ & $0.2 \%$ or $2 \mathrm{mg} / \mathrm{d}$ & $0.2 \%$ or $2 \mathrm{mg} / \mathrm{d}$ & \\
\hline$>100 \mathrm{mg}-2 \mathrm{~g}$ & & & $0.2 \%$ or $2 \mathrm{mg} / \mathrm{d}$ \\
\hline$\leq 1 \mathrm{~g}$ & $0.1 \%$ & & \\
\hline$>1 \mathrm{~g}$ & $0.05 \%$ & & \\
\hline$>2 \mathrm{~g}$ & & $0.1 \%$ & \\
\hline
\end{tabular}

degradants in 5,10,15,20-tetra( $m$-hydroxyphenyl)chlorin ( $m$ THPC), a photodynamic therapeutic agent for cancer ${ }^{82}$ ) with, a) detection at $423 \mathrm{~nm}$ and b) positive ion ESI-MS detection. Three impurity peaks are detected in this batch of drug. Peak 1 , with the $(\mathrm{M}+\mathrm{H})^{+}$ion at $m / z 679$, is 5,10,15,20tetra( $m$-hydroxyphenyl)porphyrin ( $m$-THPP), the oxidation product of $m$-THPC $(m / z 681)$. Peak 2 has an $(\mathrm{M}+\mathrm{H})^{+}$ion at $m / z 697$, indicating hydroxylation of the $m$-THPC molecule. This compound was easily dehydrated to give an $(\mathrm{M}+\mathrm{H})^{+}$ ion at $m / z 679$ corresponding to $m$-THPP when subjected to tandem MS/MS analysis. It is therefore consistent with substitution by an $-\mathrm{OH}$ group at the reduced pyrrole ring of $m$ THPC to give $\beta$-hydroxy- $m$-THPC (Fig. 9), rather than at a double bond of a pyrrole or phenyl ring. ${ }^{83)}$ Peaks 1 and 2 are therefore degradation products caused by the oxidation of $m$ THPC.

Peak 3 is an impurity carried over from the synthetic process. It has an $(\mathrm{M}+\mathrm{H})^{+}$ion at $m / z 683$ corresponding to further reduction of $m$-THPC to give the isobacteriochlorin structure (Fig. 9). It is 5,10,15,20-tetra( $m$-hydroxyphenyl)isobacteriochlorin.

The large $m$-THPC peak (peak 4), detected at $423 \mathrm{~nm}$ was not allowed to enter the ESI-MS ion source by disconnecting the LC-MS interface just before the peak was eluted, to avoid heavy contamination of the ESI ion source by a large amount of $m$-THPC. A switching valve may also be used to divert eluent from the MS in such cases.

Analysis of Chiral Impurities in Pharmaceuticals The separation and detection of chiral impurities in pharmaceuticals are of great importance because the D-isomer of a drug can have very different pharmacological, metabolic and toxicological activity from the L-isomer.

LC-MS has been used for the analysis of chiral amino acids in the identification of chiral impurities present in diastereomeric peptide drugs. ${ }^{81)}$ The peptide is hydrolyzed and the amino acids released are derivatized with $N^{2}$-(5-fluoro2,3-dinitrophenyl)-L-alanine amide (Marfey's reagent), which converts the amino acid enatiomers into the corresponding diastereoisomers for separation and detection by HPLCMS. ${ }^{81,84)}$ The specificity of the MS detection eliminated interference from other peaks encountered when an UV detector 

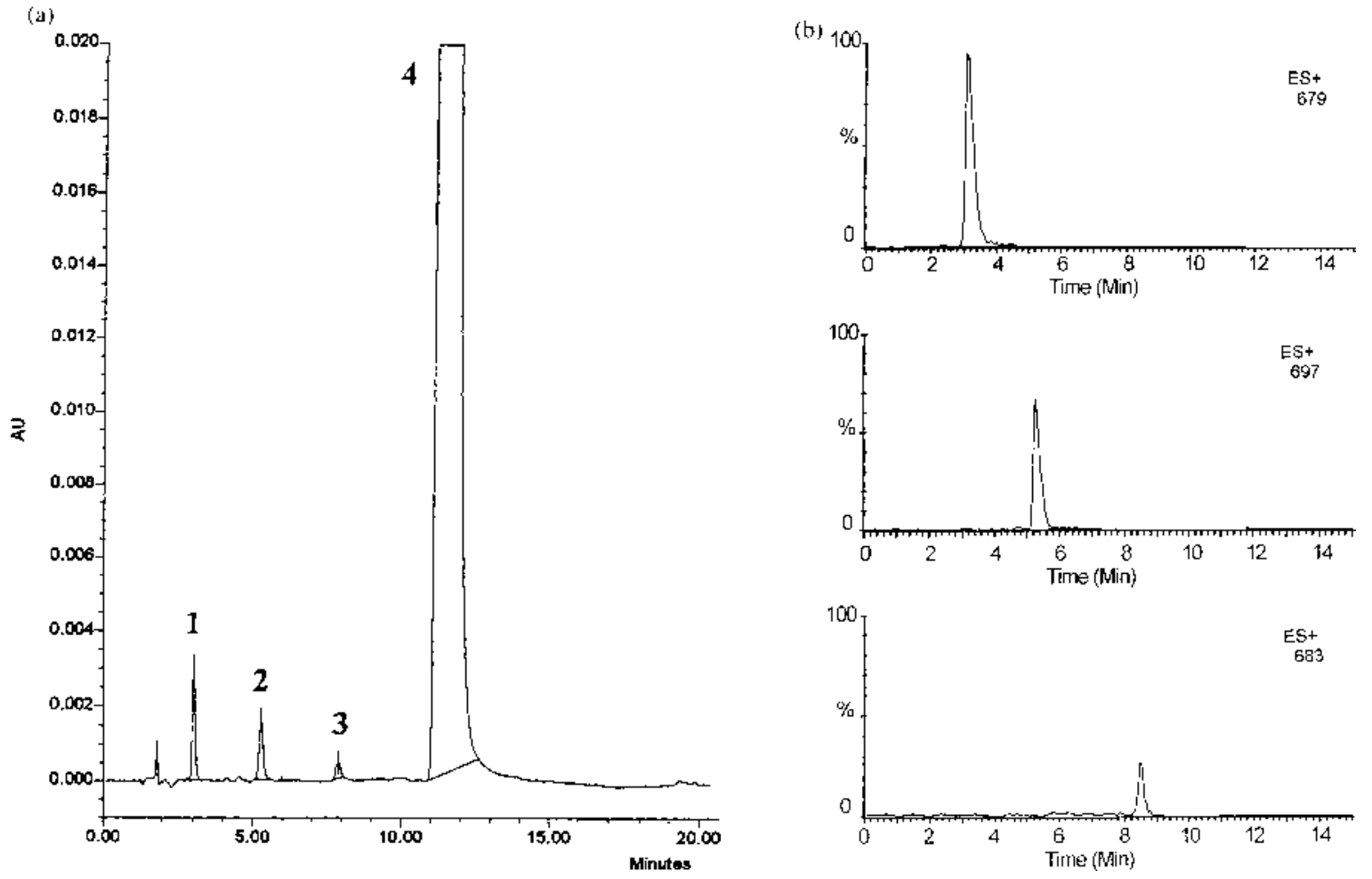

Fig. 8. Separation of Impurity and Degradation Products in a Batch of 5,10,15,20-Tetra( $m$-hydroxyphenyl)chlorin $(m$ THPC)

The separation was carried out on a Hypersil-BDS column ( $3 \mu \mathrm{m}$ particle size, $100 \times 4.6 \mathrm{~mm}$ i.d.) by gradient elution from $50 \%$ A $(0.1 \%$ TFA in water): $50 \%$ B $(0.1 \%$ TFA in acetonitrile) to $100 \%$ B in $15 \mathrm{~min}$. Detection: a), $423 \mathrm{~nm}$ and b), positive ion ESI-MS. Peaks 1, 5,10,15,20-tetra( $m$-hydroxyphenyl)porphyrin $(m$ THPP); $2, \beta$-hydroxy- $m$ THPC; 3 , 5,10,15,20-tetra( $m$-hydroxyphenyl)isobacteriochlorin; $4, m$ THPC
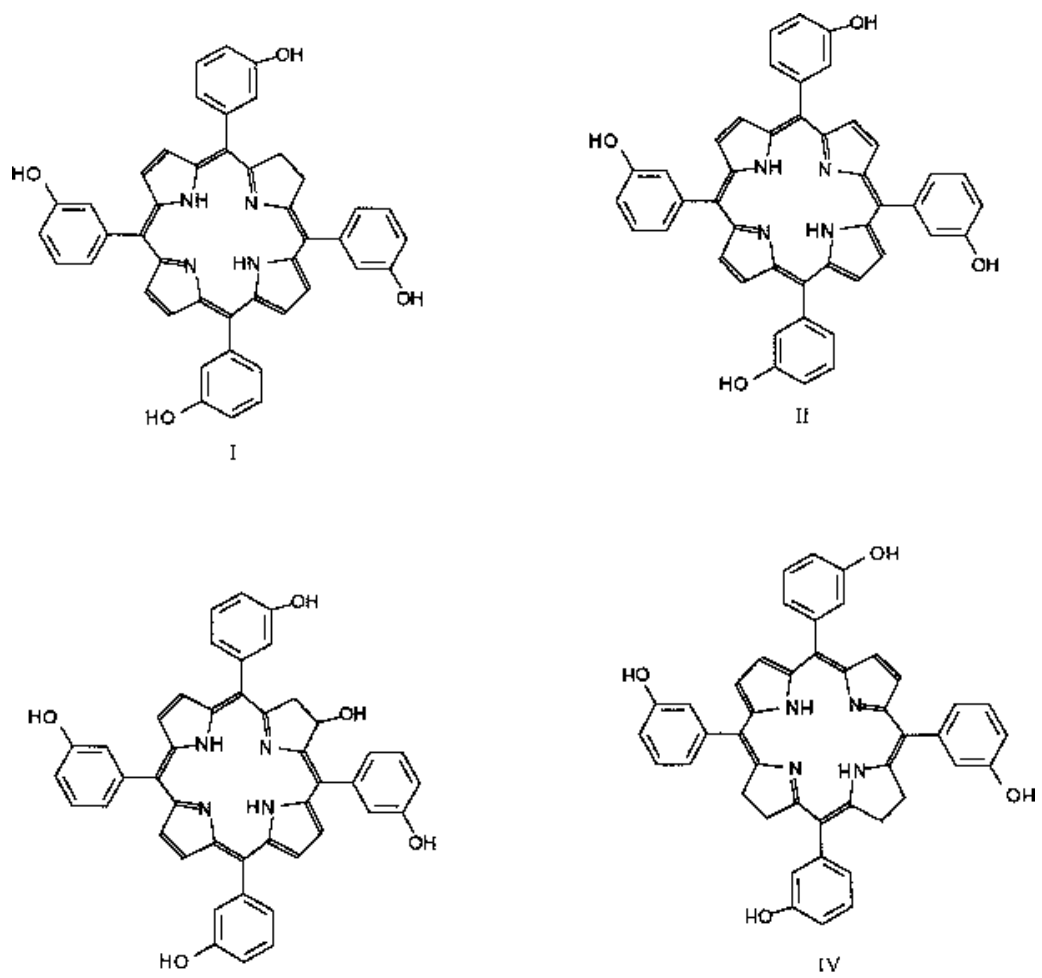

Fig. 9. Structures of $m$ THPC and Its Impurity and Degradation Products I, $m$ THPC; II, $m$ THPP; III, $\beta$-hydroxy- $m$ THPC; IV, isobacteriochlorin. 
was used for detection

Diastereoisomeric derivatization followed by LC-MS separation and detection is also useful for the analysis of chiral acidic drugs e.g. 2-arylpropionic acids. Although chiral stationary phases can be used for the enantiomeric separation of these drugs, the sensitivity of detection by negative ion ESIMS was poor. ${ }^{85)}$ They have been derivatized with benzofuran fluorescent reagents for enatiomeric separation by HPLC with fluorometric detection. ${ }^{85}$ These benzofuran derivatives are also ideal for sensitive and specific detection by positive ion ESI-MS without interference.

Diastereomeric derivatization with a suitable reagent that confers favourable MS properties to the molecules is a technique worth exploring for chiral drug analysis.

\section{FUTURE TRENDS AND PERSPECTIVES}

LC-MS has proved to be an extremely sensitive and specific technique for the analysis of pharmaceuticals. It plays important roles in the studies of drug metabolism, ${ }^{86)}$ discovery of new drug candidates and the analysis, identification and characterization of impurities and degradants in drug substances and products.

Technical advances in MS continue, with improvements in sensitivity and resolution. The trend is towards the further development of hybrid instruments such as Q-TOF. FT-ICR will become more prominent as developments are made and instruments become less complex and more available. The likely importance of proteomics in pharmaceutical development will have implications for MS, leading to the further requirements for high resolution sequencing.

Coupling high-throughput sample preparation techniques with multiplexed LC-MS-MS will lead to even faster analysis and the potential of interfacing LC-NMR with MS to give an LC-NMR-MS system will allow the unequivocal identification of drugs and metabolites. ${ }^{87,88)}$

The technical difficulties in linking micro and capillary separation techniques with nanospray MS are being solved and advances in this front can be expected. The use of microfluidic systems offers prospects for miniaturized chip separations and even the possibility of miniaturized mass spectrometers in the rather more distant future. The capabilities and expertise of Japan in miniaturized electronics on chips may be directed in this area.

\section{REFERENCES}

1) Barber M., Bordoli R. S., Sedgwick R. D., Tyler A. N., J. Chem. Soc. Chem. Commun., 1981, 325-327 (1981).

2) Scott R. P. W., Scott C. G., Karpov G. V., Munroe M., Hess J., J. Chromatogr., 99, 395-405 (1974).

3) McFadden W. H., Schwartz H. L., Evans S., J. Chromatogr., 122, 389-396 (1976).

4) Baldwin M. A., McLafferty F. W., Org. Mass Spectrom., 7, 13531356 (1973).

5) Arpino P. J., Baldwin M. A., McLafferty F. W., Biomed. Mass Spectrom., 1, 80-82 (1974).

6) Caprioli R. M., Fan T., Cottrell J. S., Anal. Chem., 58, 2949-2954 (1986).

7) Vestal M. L., Mass Spectrom. Rev., 10, 53-58 (1983).

8) Winkler P. C., Perkins D. D., Williams D. K., Browner R. F., Anal. Chem., 60, 489-495 (1988).

9) Dole M., Mach L. L., Hines R. L., Mobley R. C., Ferguson L. D., Alice M. B., J. Chem. Phys., 49, 2240-2249 (1968).
10) Whitehouse C. M., Dreyer R. N., Yamashita M., Fenn J. B., Anal. Chem., 57, 675-679 (1985).

11) Bruins A. P., Covey T. R., Henion J. D., Anal. Chem., 59, 2642-2644 (1987).

12) Banks J. F., Quinn J. P., Whitehouse C. M., Anal. Chem., 66, 3688 3695 (1994).

13) Cole R. B. (ed.), "Electrospray Ionisation Mass Spectrometry-Fundamentals, Instrumentation and Applications," Wiley, New York, 1997.

14) Gaskell S. J., J. Mass Spectrom., 32, 677-688 (1997).

15) Guiochon G., Colin H., "Proceedings of the 2nd International Congress on Techniques in Environmental Chemistry," ed. by Albaiges J., Barcelona, Spain, Nov. 1981, Pergamon Press, Oxford, 1982, pp. $169-175$.

16) Covey T., "Biological and Biotechnological Applications of Electrospray Ionisation Mass Spectrometry," ed. by Snyder A. P., ACS Symposium Series 619, American Chemical Society, Washington, DC, 1996, pp. 21-59.

17) Chowdury S. K., Chait B. T., Anal. Chem., 63, 1660-1664 (1991).

18) Gale D. C., Smith R. D., Rapid Commun. Mass Spectrom., 7, $1017-$ 1021 (1993).

19) Emmett M. R., Caprioli R. M., J. Am. Soc. Mass Spectrom., 5, 605613 (1994).

20) Wilm M. S., Mann M., Int. J. Mass Spectrom. Ion processes, 136, 167-180 (1994).

21) Wilm M. S., Mann M., Anal. Chem., 68, 1-8 (1996).

22) Xue Q., Dunayevskiy Y. M., Foret F., Karger B. L., Rapid Commun. Mass Spectrom., 11, 1253-1256 (1997).

23) Ramsey R. S., Ramsey J. M., Anal. Chem., 69, 1174-1178 (1997).

24) Figeys D., Ning Y., Abersold R., Anal. Chem., 69, 3153-3160 (1997).

25) Licklider L., Wang X.-Q., Deasi A., Tai Y.-C., Lee T. D., Anal. Chem., 72, 367-375 (2000).

26) de Mello A. J., Lab. on a Chip, 1, 7N-12N (2001).

27) Apffel A., Fischer S., Goldberg G., Goodley P., Kuhlmann F., "Proceedings of the 42nd ASMS Conference on Mass Spectrometry and Allied Topics," Chicago, IL., 1994, pp. 772-773.

28) Kuhlmann F. E., Apfel A., Fischer S. M., Goldberg G., Goodley P. C., J. Am. Soc. Mass Spectrom., 6, 1221-1225 (1995).

29) New A. P., Wolff J. C., Crabtree S., Freitas dos Santos L., Okafo G., Lee J., Divan K., J. Chromatogr. A, 913, 205-208 (2001).

30) Syage J. A., Evans M. D., Hanold K. A., Amer. Lab., 32, 42-45 (2000).

31) Robb D. B., Covey T. R., Bruins A. P., Anal. Chem., 72, 3653-3659 (2000).

32) Cappiello A., Famiglini G., Mangani F., Palma P., Mass Spectrom. Rev., 20, 88-104 (2001).

33) Stevenson T. L., Loo J. A., Greis K. D., Anal. Biochem., 262, 99-109 (1998).

34) Gusev A. I., Fresenius J. Anal. Chem., 366, 691-700 (2000).

35) Smith R. D., Barinaga C. J., Usdeth H. R., Anal. Chem., 60, 1948 1952 (1988).

36) Von Bocke A., Nicholson G., Bayer E., Electrophoresis, 22, 12511266 (2001).

37) Verheij E. R., Tjaden U. R., Niessen W. M. A., van der Greef J., J. Chromatogr., 554, 339-349 (1991).

38) Gordon D. B., Lord G. A., Jones D. S., Rapid Commun. Mass Spectrom., 8, 544-548 (1994).

39) Bartle K. D., Myers P., J. Chromatogr. A, 916, 3-23 (2001).

40) Bartle K. D., Myers P. (eds.), "Capillary Electrochromatography," RSC Chromatography Monographs, Royal Society of Chemistry, Cambridge, 2001.

41) Guilhaus M., Selby D., Mlynski V., Mass Spectrom. Rev., 19, 65-107 (2000).

42) Yang L., Wu N., Clement R. P., Rudewicz P. J., "Presentation at the 15th International Mass Spectrometry Meeting," Barcelona, 27th Aug.-1st. Sept. 2000.

43) Costello C. E., Curr. Opinion in Biotechnol., 10, 22-28 (1999).

44) Shen Y., Zhao R., Belov M. E., Conrads T. P., Anderson G. A., Tang K., Pasa-Tolic L., Veenstra T. D., Lipton M. S., Usdeth H. R., Smith R. D., Anal. Chem., 73, 1766-1775 (2001).

45) Shen Y., Tolic N., Zhao R., Pasa-Tolic L., Li L., Berger S. J., Harkewicz R., Andeson G. A., Belov M. E., Smith R. D., Anal. Chem., 73, 3011-3021 (2001).

46) De Biasi V., Haskins N., Organ A., Bateman R., Giles K., Jarvis S., Rapid Comm. Mass Spectrom., 13, 1165-1168 (1999). 
47) Eckers C., Wolff J. C., Haskins N., Sage A. B., Giles K., Bateman R., Anal. Chem., 72, 3683-3688 (2000).

48) Van Pelt C. K., Corso T. N., Schultz G. A., Lowes S., Hennion J., Anal. Chem., 73, 582-588 (2001).

49) Liu H., Felten Q. X., Zhang B., Jedrzejewski P., Karger B. L., Foret F., Anal. Chem., 72, 3303-3310 (2000).

50) Cai H., Wang Q., Luo J. L., Lim C. K., Biomed. Chromatogr., 13, 354-359 (1999).

51) Fernandez-Metzler C. L., Owens K. G., Baillie T. A., King R. G., Drug Metab. Dispos., 27, 32- 40 (1999).

52) Kato K., Jingu S., Ogawa N., Higuchi S., Biomed. Chromatogr., 16, 25-30 (2002).

53) Bruning P. F., Eur. J. Cancer, 28A, 1404-1407 (1992).

54) Poon G. K., Walter, B., Lonning P. E., Horton M. N., McCaque R., Drug. Metab. Dispos., 23, 377-382 (1995).

55) Lim C. K., Yuan Z-X., Jones R. M., White I. N. H., Smith L. L., J. Pharm. Biomed. Anal., 15, 1335-1342 (1997).

56) Ireson C., Orr S., Jones D. J. L., Verschoyle R., Lim C. K., Luo J. L., Howell L., Plummer S., Jukes R., Williams R., Steward M., Gescher A., Cancer Res., 61, 1058-1064 (2001).

57) Kyranos J. N., Cai H., Wei D., Goetzinger W. K., Curr. Opin. Biotechnol., 12, 105-111 (2001).

58) Allanson S. P., Biddlecombe R. A., Jones A. E., Pleasance S., Rapid Commun. Mass Spectrom., 10, 811—818 (1996).

59) Simpson H., Berthemy A., Buhrman D., Burton R., Newton J., Kealy M., Well D., Wu D., Rapid Commun. Mass Spectrom., 12, 75-82 (1998).

60) Zhang H., Henion J., Anal. Chem., 71, 3955-39 (1999).

61) Jemal M., Huang M., Mao Y., Whigan D., Schuster A., Rapid Commun. Mass Spectrom., 14, 1023-1028 (2000).

62) Jemal M., Biomed. Chromatogr., 14, 422-429 (2000).

63) Jemal M., Teitz D., Quyang Z., Khan S., J. Chromtogr. B, 732, 501508 (1999).

64) Zweigenbaum J., Heinig K., Steinborner S., Wachs T., Henion J., Anal. Chem., 71, 2294-2300 (1999).

65) Heinig K., Henion J., J. Chromatogr. B, 732, 445-458 (1999).

66) Wu J.-T., Zeng H., Qian M., Brogdon B. L., Unger S. E., Anal. Chem., 72, 61-67 (2000).

67) Onarato J. M., Henion J. D., Lefebure P. M., Kiplinger J. P., Anal.
Chem., 73, 119-125 (2001).

68) Peng S. X., Hansen C., Strojnowski M. J., Golebiowski A., Klopfenstein S. R., Anal. Chem., 72, 261-266 (2000).

69) Clarke S. D., Hill H. M., Noctor T. A. G., Thomas D., Pharm. Sci., 2 , 203-207 (1996).

70) Bonfiglio R., King R. C., Olah T. V., Merkle K., Rapid Commun. Mass Spectrom., 13, 1175-1185 (1999).

71) Matuszewski B. K., Constanzer M. L., Charez-Eng C. M., Anal. Chem., 70, 882-889 (1998).

72) Ayrton J., Dear G. J., Leaven W. J., Mallett D. N., Plumb R. S., J. Chromatogr. A, 828, 199-207 (1998).

73) Jemal M., Xia Y. Q., Whigan D. B., Rapid Commun. Mass Spectrom., 12, 1389-1399 (1998).

74) Jemal M., Ouyang Z., Xia Y. Q., Powell M. L., Rapid Commun. Mass Spectrom., 13, 1462-1471 (1999).

75) Wu J. T., Zeng H., Qian M., Brogdon B. L., Unger S. E., Anal. Chem., 72, 61-67 (2000).

76) Xia Y. Q., Whigan D. B., Powell M. L., Jemal M., Rapid Commun. Mass Spectrom., 14, 105-111 (2000).

77) Volk K, J., Klohr S. E., Rourick R. A., Kerns E. H., Lee M. S., J. Pharm. Biomed. Anal., 14, 1663-1674 (1996).

78) Wu Y., Biomed. Chromatogr., 14, 384-396 (2000).

79) Eckers C., Haskins N., Langridge J., Rapid Commun. Mass Spectrom., 11, 1916-1922 (1997).

80) Ermer J., J. Pharm. Biomed. Anal., 18, 707-714 (1998).

81) Ermer J., Vogel M., Biomed. Chromatogr., 14, 373-383 (2000).

82) Stewart J. C. M., "New Approaches to Cancer Treatment," ed. by Horrobin D. F., Churchill Communications Europe, London, 1994, pp. $109-120$.

83) Jones R. M., Wang Q., Lamb J. H., Djelal B. D., Bonnett R., Lim. C. K., J. Chromatogr. A, 722, 257-265 (1996).

84) Fujii K., Ikai Y., Oka H., Suzuki M., Harada K., Anal. Chem., 69, 3346-3352 (1997).

85) Santa T., Luo J., Lim C. K., Imai K., Biomed. Chromatogr., 12, 73-77 (1998).

86) Oliveira E. J., Watson D. G., Biomed. Chromatogr., 14, 351-372 (2000).

87) Peng S. X., Biomed. Chromatogr., 14, 430-441 (2000).

88) Wilson I. D., J. Chromatogr., 892, 315-327 (2000). 